\title{
On Realization of the Bose-Einstein Condensates and Quantum Superfluids
}

\begin{abstract}
T. DOMAŃski
Institute of Physics, M. Curie-Skłodowska University, pl. M. Curie-Skłodowskiej 1, 20-031 Lublin, Poland

The purpose of this work is twofold. In the first part we describe superfluidity/superconductivity as an emergent phenomenon resulting from two-body correlations in presence of the Bose-Einstein condensation of particles. We briefly discuss the underlying mechanism for bosons as well as fermion pairs and illustrate various realizations of superfluidity emphasizing the recent examples. In the second part we study the glassy liquid of incoherent pairs which might exist above the transition temperature $T_{\mathrm{c}}$ in the underdoped regime of cuprate superconductors. In particular, we explore the angular variation of pseudogap within two-dimensional version of the boson-fermion model using for a quantitative analysis the projective method. We find that above $T_{\mathrm{c}}$ the pseudogap closes first near the nodal areas restoring there pieces (arcs) of the Fermi surface whereas remaining parts of the large Fermi surface around the antinodal points are still absent due to incoherent pairs. Upon increasing temperature the length of the Fermi arcs enlarges because the superconducting correlations are gradually suppressed. An intriguing death of Fermi surface can thus be closely related to the Bogoliubov quasiparticles whose existence in the pseudogap state has been predicted by us and confirmed recently by the angle resolved photoemission spectroscopy measurements on $\mathrm{Bi}_{2} \mathrm{Sr}_{2} \mathrm{CaCu}_{2} \mathrm{O}_{8}$ and $\mathrm{La}_{1.895} \mathrm{Sr}_{0.105} \mathrm{CuO}_{4}$ compounds.
\end{abstract}

PACS numbers: 74.20.-z, 74.20.Mn, 74.40.-n

\section{Preliminaries}

Quantum statistical mechanics puts a strong emphasis on the crucial distinction between fermions obeying the Pauli exclusion principle and bosons having no upper bounds for occupying any quantum state at hand. Under specific conditions the latter can macroscopically accommodate in the lowest energy level (for instance in $\boldsymbol{q}=\mathbf{0}$ momentum of the noninteracting uniform gas or the zero frequency oscillator level $E_{0}=\frac{1}{2} \hbar \omega$ for the case of magneto-optically trapped atoms). This phenomenon, referred to as the Bose-Einstein condensation (BEC), has been discovered for the first time in the superfluid state of ${ }^{4} \mathrm{He}$. At temperatures far below the lambda transition about $8 \%$ of helium atoms gets frozen to the condensate. Recently, the development of trapping and cooling techniques enabled production of the artificial condensates for several atoms. Very dilute densities of the trapped atoms $\approx 10^{14} \mathrm{~cm}^{-3}$ require that the quantum degeneracy level is reached at ultracold temperatures, below $\mu \mathrm{K}$. Such stringent conditions have been so far achieved by a dozen of groups worldwide and the presently available experimental conditions allow for condensation of more than $90 \%$ of the trapped atoms [1].

A direct realization of BEC among fermions is obviously prohibited. However, an equivalent phenomenon can be obtained in two steps. First, one needs to combine the initial fermions (at least some of their states located near the Fermi level) into the pairs (composite bosons). Depending on specific fermions the possible mechanisms can range from e.g. the phonon mediated pairing in classical superconductors to any other effective attractions - some examples will be discussed in Sect. 2.3. In the second step, these fermion pairs brought to a single state can become coherent showing up on the macroscopic level a loss of their resistance etc.

The distinct statistical character of fermions and bosons can be, sort of, unified by realization of their superfluid/superconducting states. A deeper support for this analogy has been given by Leggett (for a comprehensive discussion see Sect. 4.4 in Ref. [1]) who noticed that structures of the ground state wave function in the $\mathrm{BEC}$ of bosons and the BCS state of fermion pairs are identical. A controlled realization of a crossover between the bosonic and fermionic condensates (and their superfluid states) is presently attainable using the mixtures of ultracold fermion atoms [2]. This fast developing discipline might eventually serve as a testing field also for the solid state physics, in particular clarifying the theoretical aspects of high temperature superconductivity. One of them is the questionable issue of incoherent fermion pairs possibly existing above $T_{\mathrm{c}}$ in the underdoped regime, as discussed in Sect. 3.

\section{Basic remarks on superfluidity}

\subsection{Mechanism of the boson superfluidity}

Although BEC is crucial for transition to the superfluid state, yet such condition alone is not sufficient. Another necessary ingredient is the long range coherence which can eventually set on due to the correlations. To explain this point let us consider the Hamiltonian of interacting bosons

$$
\hat{H}=\int \mathrm{d} \boldsymbol{r} \hat{\Psi}^{\dagger}(\boldsymbol{r}, t)\left(-\frac{\hbar^{2}}{2 m} \nabla^{2}+V_{\text {ext }}(\boldsymbol{r})-\mu\right) \hat{\Psi}(\boldsymbol{r}, t)
$$




$$
\begin{aligned}
& +\frac{1}{2} \int \mathrm{d} \boldsymbol{r} \mathrm{d} \boldsymbol{r}^{\prime} \hat{\Psi}^{\dagger}(\boldsymbol{r}, t) \hat{\Psi}^{\dagger}\left(\boldsymbol{r}^{\prime}, t\right) U\left(\boldsymbol{r}-\boldsymbol{r}^{\prime}\right) \\
& \times \hat{\Psi}\left(\boldsymbol{r}^{\prime}, t\right) \hat{\Psi}(\boldsymbol{r}, t) .
\end{aligned}
$$

If a fraction of particles is Bose-Einstein (BE) condensed, then the field operators $\hat{\Psi}(\boldsymbol{r}, t)$ can be decomposed into

$$
\hat{\Psi}(\boldsymbol{r}, t)=\Phi(\boldsymbol{r}, t)+\delta \hat{\Psi}(\boldsymbol{r}, t) .
$$

where $\Phi(\boldsymbol{r}, t)$ describes macroscopic function of the condensate and $\delta \hat{\Psi}(\boldsymbol{r}, t)$ refers to the non-condensed bosons.

Substituting (2) to the Heisenberg equation and assuming the point-like scattering potential $V\left(\boldsymbol{r}-\boldsymbol{r}^{\prime}\right)=$ $g \delta\left(\boldsymbol{r}-\boldsymbol{r}^{\prime}\right)$ one obtains the following Gross-Pitaevskii (GP) equation [3]:

$$
\begin{aligned}
& \mathrm{i} \hbar \frac{\partial \Phi(\boldsymbol{r}, t)}{\partial t} \\
&=\left[-\frac{\hbar^{2} \nabla^{2}}{2 m}+V_{\text {ext }}(\boldsymbol{r})+g n_{\mathrm{c}}(\boldsymbol{r}, t)-\mu\right] \Phi(\boldsymbol{r}, t),
\end{aligned}
$$

which describes the nonuniform and dynamic BE condensate fraction $n_{\mathrm{c}}(\boldsymbol{r}, t)=|\Phi(\boldsymbol{r}, t)|^{2}$. Solution of the nonlinear GP equation (3) is not feasible by other than numerical means, but we can point out some of its important consequences without resorting to the numerics.

Complex function $\Phi(\boldsymbol{r}, t)$ of the condensate can be represented through its amplitude $|\Phi(\boldsymbol{r}, t)|$ and phase $\theta(\boldsymbol{r}, t)$

$$
\Phi(\boldsymbol{r}, t)=\sqrt{n_{\mathrm{c}}(\boldsymbol{r}, t)} \mathrm{e}^{\mathrm{i} \theta(\boldsymbol{r}, t)} .
$$

Using these variables, the density of superfluid current $\boldsymbol{j}_{\mathrm{c}}(\boldsymbol{r}, t) \equiv \frac{\hbar}{2 \mathrm{i}}\left(\Phi^{*} \nabla \Phi-\Phi \nabla \Phi^{*}\right)$ can be expressed as

$$
\boldsymbol{j}_{\mathrm{c}}(\boldsymbol{r}, t)=\hbar n_{\mathrm{c}}(\boldsymbol{r}, t) \nabla \theta(\boldsymbol{r}, t) .
$$

Equation (4) implies that the supercurrent is irrotational

$$
\nabla \times \boldsymbol{j}_{\mathrm{c}}(\boldsymbol{r}, t)=0
$$

unless singularities of the phase $\theta(\boldsymbol{r}, t)$ are encountered. Such type of topological defects leads to formation of the quantized vortices which geometrically arrange themselves into the Abrikosov lattice [1,3].

Rewriting (3) for the amplitude and phase one obtains the following coupled equations:

$$
\begin{aligned}
& \frac{\partial}{\partial t} n_{\mathrm{c}}(\boldsymbol{r}, t)+\nabla \cdot \boldsymbol{j}_{\mathrm{c}}(\boldsymbol{r}, t)=0 \\
& m\left(\frac{\partial \boldsymbol{v}_{\mathrm{c}}(\boldsymbol{r}, t)}{\partial t}+\nabla \frac{v_{\mathrm{c}}^{2}(\boldsymbol{r}, t)}{2}\right)=-\nabla\left(V_{\mathrm{eff}}+g n_{\mathrm{c}}-\mu\right)
\end{aligned}
$$

where $V_{\text {eff }}(\boldsymbol{r}, t)=V_{\text {ext }}(\boldsymbol{r})-\frac{\hbar^{2}}{2 m \sqrt{n_{\mathrm{c}}(\boldsymbol{r}, t)}} \nabla^{2} \sqrt{n_{\mathrm{c}}(\boldsymbol{r}, t)}$ and the superfluid velocity given by $\boldsymbol{v}_{\mathrm{c}}(\boldsymbol{r}, t)=\frac{\hbar}{m} \nabla \theta(\boldsymbol{r}, t)$. The set of Eqs. (6), (7) is identical with the classical hydrodynamics for an ideal liquid which has no viscosity. In particular, the continuity constraint is expressed by (6) and the, so-called, Josephson equation (7) reproduces the Euler equation.

Hydrodynamic approach described above (which is well justified for the collisionless limit) proves that indeed BEC along with the correlations constitute the superfluidity. The corresponding phase transition itself is of the second order-type and is related to the symmetry break- ing caused by appearance of the off-diagonal long-range order (ODLRO) which in the present context comes from the nonvanishing expectation value $\langle\hat{\Psi}(\boldsymbol{r}, t)\rangle=\Phi(\boldsymbol{r}, t)$.

\subsection{Superfluidity of the fermion pairs}

To comment on the BEC and superfluidity of fermions in some analogy to discussion of the preceding section let us consider the mixture of spin $\sigma=\uparrow$ and $\downarrow$ particles

$$
\begin{aligned}
\hat{H} & =\int \mathrm{d} \boldsymbol{r} \sum_{\sigma} \hat{c}_{\sigma}^{\dagger}(\boldsymbol{r}, t)\left[-\frac{\hbar^{2} \nabla^{2}}{2 m}+V_{\mathrm{ext}}(\boldsymbol{r})-\mu\right] \hat{c}_{\sigma}(\boldsymbol{r}, t) \\
& +\frac{1}{2} \int \mathrm{d} \boldsymbol{r} \mathrm{d} \boldsymbol{r}^{\prime} \hat{c}_{\uparrow}^{\dagger}(\boldsymbol{r}, t) \hat{c}_{\downarrow}^{\dagger}\left(\boldsymbol{r}^{\prime}, t\right) U\left(\boldsymbol{r}-\boldsymbol{r}^{\prime}\right) \\
& \times \hat{c}_{\downarrow}\left(\boldsymbol{r}^{\prime}, t\right) \hat{c}_{\uparrow}(\boldsymbol{r}, t)
\end{aligned}
$$

interacting by an attractive potential $U\left(\boldsymbol{r}-\boldsymbol{r}^{\prime}\right)<0$ whose origin can be arbitrary. "Spins" denote symbolically the quantum numbers, for instance in the case of ultracold atoms trapped by potential $V_{\text {ext }}(\boldsymbol{r})$ corresponding to the hyperfine configurations, for the confined quarks to colors, for charge carriers in the solids either to electron spins, orbital labels, or any other degrees of freedom.

A role similar to the boson operators (2) is for fermion systems played by the pairing field

$$
\hat{\pi}(\boldsymbol{r}, t) \equiv \int \mathrm{d} \boldsymbol{r}^{\prime} \hat{c}_{\downarrow}\left(\boldsymbol{r}+\frac{1}{2} \boldsymbol{r}^{\prime}, t\right) \hat{c}_{\uparrow}\left(\boldsymbol{r}-\frac{1}{2} \boldsymbol{r}^{\prime}, t\right) .
$$

If a macroscopic number of fermion pairs occupies the single, lowest energy state we can decompose $\hat{\pi}(\boldsymbol{r}, t)$ into the condensate fraction and a remaining part corresponding to "excited" pairs

$$
\hat{\pi}(\boldsymbol{r}, t)=\langle\hat{\pi}(\boldsymbol{r}, t)\rangle+\delta \hat{\pi}(\boldsymbol{r}, t) .
$$

The complex order parameter $\langle\hat{\pi}(\boldsymbol{r}, t)\rangle \equiv \chi(\boldsymbol{r}, t)$ is responsible for ODLRO which is signified by non-vanishing asymptotics $\lim _{\left|\boldsymbol{r}_{1}-\boldsymbol{r}_{2}\right| \rightarrow \infty}\left\langle\hat{\pi}\left(\boldsymbol{r}_{1}, t\right) \hat{\pi}\left(\boldsymbol{r}_{2}, t\right)\right\rangle=$ $\chi^{*}\left(\boldsymbol{r}_{1}, t\right) \chi\left(\boldsymbol{r}_{2}, t\right) \neq 0$. As far as the "excited" fermion pairs are concerned they are nearly irrelevant for a behavior of the superfluid fraction. However, their presence might show up indirectly affecting the single particle spectrum by depleting the low energy states or bringing the anomalous contributions to the diamagnetic as well as charge response, especially near $T_{\mathrm{c}}$.

For a quantitative determination of the superfluid fraction $n_{\mathrm{c}}(\boldsymbol{r}, t)=|\chi(\boldsymbol{r}, t)|^{2}$ and the supercurrent one needs to apply some approximations, because the Hamiltonian (8), quartic in the fermion operators, cannot be analytically solved. The simplest possible treatment relies on the Bogoliubov-de Gennes (BdG) ansatz

$$
\begin{aligned}
& \hat{c}_{\uparrow}(\boldsymbol{r}, t)=\sum_{n}\left[u_{n}(\boldsymbol{r}, t) \hat{\tilde{c}}_{\uparrow, n}+v_{n}(\boldsymbol{r}, t) \hat{\tilde{c}}_{\downarrow, n}^{\dagger}\right], \\
& \hat{c}_{\downarrow}^{\dagger}(\boldsymbol{r}, t)=\sum_{n}\left[-v_{n}^{*}(\boldsymbol{r}, t) \hat{\tilde{c}}_{\uparrow, n}+u_{n}^{*}(\boldsymbol{r}, t) \hat{\tilde{c}}_{\downarrow, n}^{\dagger}\right],
\end{aligned}
$$

where the complex coefficients $u_{n}(\boldsymbol{r}, t)$ and $v_{n}(\boldsymbol{r}, t)$ can be estimated requiring diagonal structure for the Hamiltonian (8) expressed in the new operators $\hat{\tilde{c}}_{\sigma, n}^{(\dagger)}$. This $\mathrm{BdG}$ procedure has the mean field character neglecting quantum fluctuations which are explicitly pointed out in Sect. 3.1. They can be partly incorporated through a 
perturbative expansion around the saddle point but such analysis is rather cumbersome. In the next section we will sketch an alternative method which seems to be suitable for the resonant Feshbach-type interactions.

Substituting the ansatz (11), (12) to the pair operator (9) one obtains the spatially and time dependent order parameter $\chi(\boldsymbol{r}, t)[2]$. It can be analytically shown [4] that this BdG procedure (11), (12) is fully equivalent to the Gross-Pitaevskii equation (3) applied to the macroscopic function $\chi(\boldsymbol{r}, t)$. We hence conclude that BEC of the correlated paired fermions leads to their dissipationless flow accompanied by other features of the superfluid state such as the quantized vortices, collective sound-wave modes, Josephson effect etc.

\subsection{Survey of the quantum superfluids}

There are known various examples of BEC and their corresponding quantum superfluids. Here we point out several realizations for the bosonic species.

a) Historically the first superfluid state has been discovered for ${ }^{4} \mathrm{He}$ below the lambda temperature $T_{\lambda} \approx 2.17 \mathrm{~K}$. Interactions between helium atoms are however so strong that $\mathrm{BE}$ condensate is considerably depleted and also the lifetimes of the quasiparticles are strongly suppressed.

b) The exotic form of superfluidity can be achieved for spins in the ferromagnetic materials, where the elementary excitations (magnons) obey bosonic statistics. The available experimental techniques allow for a control of these quanta population (at least over time scale on the order of seconds) so that the magnon thermalization and formation of BEC are possible. The emerging spin superfluidity manifests itself by coherent transport of the magnetization over macroscopic distances [5].

c) In semiconductors the conduction band electrons do form the bound states (excitons) with the valence band holes. These bosonic objects could undergo the BE condensation, however some obstacles arise due to their short lifetimes and very diluted concentrations. The experimental signatures of excitonic BEC has been recently reported using the coupled quantum wells in the quasi-two-dimensional heterostructures of GaAs/AgAlAs [6].

d) Enormous activity has been also focused on producing the condensates using the dilute quantum gases of atoms in the magneto-optical traps (for a list of bosonic atoms see e.g. Ref. [7]). Experimental detection of BEC is done in such systems by means of the time of flight measurements. Despite the extremely low temperatures, there can be condensed beyond $90 \%$ of the trapped atoms (ten times more than in ${ }^{4} \mathrm{He}$ ). A more difficult problem is to see the superfluid features. So far the most popular way was based on observation of quantum vortices [8] but later also the dc and ac Josephson effects were experimentally mastered [9].
Superfluidity of the composite bosons such as fermion pairs can occur in plenty of situations. Let us list here some representative examples.

a) In classical superconductors the phonon-mediated attraction induces the Cooper pairs defined in $\boldsymbol{k}$-space between electrons on very large distances. Appearance of these pairs is therefore simultaneously accompanied by the onset of ODLRO which on macroscopic level yields the ideal dc conductance and perfect diamagnetism.

b) Superfluid transition of the correlated charge carriers in the high temperature superconductors has a completely different character. Proximity of the superconducting dome to the Mott insulator indicates that the paring mechanism of cuprate oxides is non-retarded. It probably originates from the sole Coulomb repulsion which effectively induces the antiferromagnetic exchange [10] leading to the intersite $d$-wave pairing. Besides this rather obvious and widely accepted scenario there are still many controversies with regard to the pseudogap state above $T_{\mathrm{c}}$. One of popular interpretations assigns it to the preformed pairs whose long-range coherence is absent because of the small superfluid stiffness and/or other sorts of quantum fluctuations. We shall come back to this issue in the next Sect. 3.

c) Exotic forms of superconductivity can be realized in the high energy physics of quarks. Since quarks strongly attract each other, expectation of their color superfluidity seems to be natural [11]. Masses of the confined quarks differ a lot, therefore there could appear certain unusual kinds of superfluidity such as the Larkin-Ovchinnikov-Fulde-Ferrell phase (where pairs have a non-zero total momentum) or the breached superfluidity [12] where pairing engages the light quarks from a vicinity of the Fermi energy and the heavy quarks located deep under $\varepsilon_{\mathrm{F}}$ (this, so-called, "interior" superfluidity can be further generalized on the "exterior" case).

Such two groups of superfluids are mixed with one another in the case of ultracold fermion atoms. Part of the atoms form the shallow bound states, i.e. bosonic molecules (closed channel) and the rest exists as the single fermions (open channel). Energy cost of both configurations can be varied applying the external magnetic field. In particular, when energy of the closed channel matches the open channel, there appears a strong resonant scattering [2]. This resonant Fesbhach mechanism enabled a smooth evolution between the molecular BEC and the BCS state of the open channel. Convenient tool for a description of the Feshbach resonances is the two component boson-fermion model [13]. 


\section{Pairing in the cuprates}

Measurements by the angle resolved photoemission spectroscopy (ARPES) have unambiguously established that the high temperature superconductors (HTSC) below transition temperature $T_{\mathrm{c}}$ have roughly the usual BCS properties [14]. The recent ARPES experiments $[15,16]$ provide an intriguing evidence that even in the normal state there exist the Bogoliubov-type quasiparticles typically known only for the superconductors. We have predicted such effect [17] on a basis of the phenomenological boson-fermion model which fairly well accounts for the strong pairing correlations.

Although there is no consensus on a nature of the pseudogap [18], these recent ARPES data [15, 16] and the proximity effect induced in the nanosize metallic slabs deposited on $\mathrm{La}_{2-x} \mathrm{Sr}_{x} \mathrm{CuO}_{4}$ seen for $T>T_{\mathrm{c}}$ [19] as well as the earlier studies [20] strongly support its interpretation as the precursor pairing effect. Preformed pairs are assumed to form already in the normal state but transition to the superfluidity can occur at lower temperatures upon their BE condensation establishing the long-range coherence [21]. Besides the cuprate superconductors such scenario takes place also in the ultracold atomic gases of $\mathrm{Li}^{6}$ and $\mathrm{K}^{40}[2]$. Near the Feshbach resonance the weakly bound boson molecules are scattered into the Cooper-like pairs and this unitary limit, between the BCS and BEC regimes, is affected by the strong pairing fluctuations [1].

In this section we explore the two-dimensional version of the boson-fermion model [22] for a realistic set parameters treating the itinerant fermions and their paired counterparts in the self-consistent way. We analyze the angular dependence of pseudogap and signatures of the Bogoliubov quasiparticles above $T_{\mathrm{c}}$ showing that the superconducting correlations can destroy parts of the large Fermi surface leading to death of the Fermi surface [23].

\subsection{Model for the resonant pairing}

Hamiltonian of the boson-fermion (BF) model [22] for the uniform systems is given by

$$
\begin{aligned}
& \hat{H}=\sum_{\boldsymbol{k}, \sigma}\left(\varepsilon_{\boldsymbol{k}}-\mu\right) \hat{c}_{\boldsymbol{k} \sigma}^{\dagger} \hat{c}_{\boldsymbol{k} \sigma}+\sum_{\boldsymbol{q}}\left(E_{\boldsymbol{q}}-2 \mu\right) \hat{b}_{\boldsymbol{q}}^{\dagger} \hat{b}_{\boldsymbol{q}} \\
& +\frac{1}{\sqrt{N}} \sum_{\boldsymbol{k}, \boldsymbol{q}}\left(g_{\boldsymbol{k}, \boldsymbol{q}} \hat{b}_{\boldsymbol{q}}^{\dagger} \hat{c}_{\boldsymbol{q}-\boldsymbol{k} \downarrow} \hat{c}_{\boldsymbol{k} \uparrow}+g_{\boldsymbol{k}, \boldsymbol{q}}^{*} \hat{c}_{\boldsymbol{k} \uparrow}^{\dagger} \hat{c}_{\boldsymbol{q}-\boldsymbol{k} \downarrow}^{\dagger} \hat{b}_{\boldsymbol{q}}\right),
\end{aligned}
$$

where operators $\hat{c}_{\boldsymbol{k} \sigma}^{(\dagger)}$ annihilate (create) the single fermions with energy $\varepsilon_{\boldsymbol{k}}$ and $\hat{b}_{\boldsymbol{q}}^{(\dagger)}$ correspond to the local pairs of energy $E_{\boldsymbol{q}}$. Coupling between the single and paired fermions is denoted by $g_{\boldsymbol{k}, \boldsymbol{q}}$. We assume that concentration of pairs per lattice site is small enough so that $\hat{b}_{\boldsymbol{q}}^{(\dagger)}$ can be treated as the usual boson operators (neglecting the hard-core effect). Such model (13) has been invented [22] prior to discovery of the HTCS materials and so far its properties have been analyzed by a number of groups [24].

In cuprate superconductors the itinerant charge carriers get combined into the intersite ( $d$-wave symmetry) pairs purely because of the strong on-site Coulomb repulsion between the opposite spin fermions. "Locality of pairs" should hence be understood as defined on the bonds [25] (like in the resonant valence band (RVB) theory [26]) rather than literary on the original lattice sites. However, no matter what a specific pairing mechanism is, the model (13) is useful for determination of the effective low-energy physics because the operators $\hat{b}^{(\dagger)}$ can be interpreted e.g. as the auxiliary boson fields originating from the exact Hubbard-Stratonovic transformation applied in the pairing channel to the correlated fermions. Most of the studies discussed so far in the literature have adopted various many-body techniques directly to the correlated fermions. Nonetheless, this fact stands in no conflict with the simplified two-component scenario (13). Actually it also turned out to be a very convenient tool for description of the ultracold fermion atoms resonantly interacting through the Feshbach mechanism [27].

The potential of boson-fermion interactions can be expressed in a more compact form using the following pair operators:

$$
\hat{B}_{\boldsymbol{q}}=\sum_{\boldsymbol{k}} g_{\boldsymbol{k}, \boldsymbol{q}} \hat{c}_{\boldsymbol{q}-\boldsymbol{k} \downarrow} \hat{c}_{\boldsymbol{k} \uparrow}, \quad \hat{B}_{\boldsymbol{q}}^{\dagger}=\left(\hat{B}_{\boldsymbol{q}}\right)^{\dagger},
$$

which simplify the charge exchange term to

$$
\hat{V}^{\mathrm{B}-\mathrm{F}}=\frac{1}{\sqrt{N}} \sum_{\boldsymbol{q}}\left(\hat{b}_{\boldsymbol{q}}^{\dagger} \hat{B}_{\boldsymbol{q}}+\hat{B}_{\boldsymbol{q}}^{\dagger} \hat{b}_{\boldsymbol{q}}\right) .
$$

On the mean-field level one usually approximates the interaction by its linearized structure

$$
\begin{aligned}
& \hat{V}^{\mathrm{B}-\mathrm{F}} \simeq \sum_{\boldsymbol{k}}\left[g_{\boldsymbol{k}, \mathbf{0}} \frac{\left\langle\hat{b}_{\mathbf{0}}^{\dagger}\right\rangle}{\sqrt{N}} \hat{c}_{-\boldsymbol{k} \downarrow} \hat{c}_{\boldsymbol{k} \uparrow}+\frac{1}{\sqrt{N}} \hat{b}_{\mathbf{0}}^{\dagger}\left\langle\hat{B}_{\mathbf{0}}\right\rangle+\text { H.c. }\right] \\
& -\frac{1}{\sqrt{N}}\left(\left\langle b_{\mathbf{0}}^{\dagger}\right\rangle\left\langle B_{\mathbf{0}}\right\rangle+\left\langle b_{\mathbf{0}}\right\rangle\left\langle B_{\mathbf{0}}^{\dagger}\right\rangle\right) .
\end{aligned}
$$

neglecting the contributions from

(a) finite momentum pairs $\frac{1}{\sqrt{N}} \sum_{\boldsymbol{q} \neq \mathbf{0}}\left(\hat{b}_{\boldsymbol{q}}^{\dagger} \hat{B}_{\boldsymbol{q}}+\right.$ H.c. $)$,

(b) fluctuations of condensed pairs $\frac{\delta \hat{b}_{\mathbf{0}}^{\dagger}}{\sqrt{N}} \delta \hat{B}_{\mathbf{0}}+\frac{\delta \hat{b}_{\mathbf{o}}}{\sqrt{N}} \delta \hat{B}_{\mathbf{0}}^{\dagger}$,

where $\delta \hat{b}_{\mathbf{0}}=\hat{b}_{\mathbf{0}}-\left\langle\hat{b}_{\mathbf{0}}\right\rangle$ and $\delta \hat{B}_{\mathbf{0}}=\hat{B}_{\mathbf{0}}-\left\langle\hat{B}_{\mathbf{0}}\right\rangle$.

The linearized B-F interaction (16) formally decouples the boson from fermion parts which under such conditions become exactly diagonalizable [24]. In consequence, the single particle spectrum of fermions acquires then the conventional BCS structure $A^{\mathrm{MF}}(\boldsymbol{k}, \omega)=u_{\boldsymbol{k}}^{2} \delta\left(\omega-E_{\boldsymbol{k}}\right)+$ $v_{\boldsymbol{k}}^{2} \delta\left(\omega+E_{\boldsymbol{k}}\right)$ with the Bogoliubov-type quasiparticle energy $E_{\boldsymbol{k}}=\sqrt{\left(\varepsilon_{\boldsymbol{k}}-\mu\right)^{2}+\Delta_{k}^{2}}$ and the usual coherence factors given by $u_{\boldsymbol{k}}^{2}, v_{\boldsymbol{k}}^{2}=\frac{1}{2}\left[1 \pm\left(\varepsilon_{\boldsymbol{k}}-\mu\right) / E_{\boldsymbol{k}}\right]$. The energy gap is given by $\Delta_{\boldsymbol{k}}=g_{\boldsymbol{k}, \mathbf{0}} \sqrt{\left\langle n_{\mathbf{0}}^{\mathrm{B}}\right\rangle}$ which means that fermions undergo transition to the superconducting state simultaneously with the BE condensation of bosons [22]. The latter property is exact [28] without limitations to any approximation.

Obviously, the mean-field treatment neither takes into account the fluctuations $\delta \hat{H}_{0}$ nor the finite momentum pairs $\delta \hat{H}^{\prime}$. Efficiency of such terms is expected to increase 
upon approaching $T_{\mathrm{c}}$ in the underdoped regime. We shall discuss major effects arising from these ingredients.

\subsection{Beyond the mean field framework}

To go beyond the mean field scheme there can be implemented various techniques dealing with the terms $\delta \hat{H}^{\prime}$ and $\delta \hat{H}_{0}$ mainly in a perturbative manner (see for instance [29] and other references cited therein). Here we proceed guided by the RG-like algorithm [30]. The projective character of our approach can be illustrated as follows: we propose to redefine the Bogoliubov transformation by adding new terms corresponding to scattering of fermions on the finite momentum pairs $\hat{b}_{\boldsymbol{q} \neq \mathbf{0}}^{(\dagger)}$. We presume that such pairs can exist below as well as above $T_{\mathrm{c}}$, although in the latter case the ODLRO is going to disappear because of lack of the BE condensate. We thus propose the following ansatz (its formal derivation is discussed in Sect. 3.2)

$$
\begin{aligned}
& \hat{\tilde{c}}_{\boldsymbol{k} \uparrow}=u_{\boldsymbol{k}} \hat{c}_{\boldsymbol{k} \uparrow}+v_{\boldsymbol{k}} \hat{c}_{-\boldsymbol{k} \downarrow}^{\dagger} \\
& +\frac{1}{\sqrt{N}} \sum_{\boldsymbol{q} \neq \mathbf{0}}\left(u_{\boldsymbol{k}, \boldsymbol{q}} \hat{b}_{\boldsymbol{q}}^{\dagger} \hat{c}_{\boldsymbol{q}+\boldsymbol{k} \uparrow}+v_{\boldsymbol{k}, \boldsymbol{q}} \hat{b}_{\boldsymbol{q}} c_{\boldsymbol{q}-\boldsymbol{k} \downarrow}^{\dagger}\right) \\
& \quad+\frac{1}{N} \sum_{\boldsymbol{q}, \boldsymbol{q}^{\prime} \neq \mathbf{0}}\left(u_{\boldsymbol{k}, \boldsymbol{q}, \boldsymbol{q}^{\prime}} \hat{b}_{\boldsymbol{q}}^{\dagger} \hat{b}_{-\boldsymbol{q}^{\prime}} \hat{c}_{\boldsymbol{q}+\boldsymbol{q}^{\prime}+\boldsymbol{k} \uparrow}\right. \\
& \left.\quad+v_{\boldsymbol{k}, \boldsymbol{q}, \boldsymbol{q}^{\prime}} \hat{b}_{\boldsymbol{q}} \hat{b}_{-\boldsymbol{q}^{\prime}}^{\dagger} c_{\boldsymbol{q}+\boldsymbol{q}^{\prime}-\boldsymbol{k} \downarrow}^{\dagger}\right)+\mathrm{O}\left(\hat{b}^{3}\right), \\
& \quad+\frac{1}{\sqrt{N}} \sum_{\boldsymbol{q} \neq \mathbf{0}}\left(-v_{\boldsymbol{k}, \boldsymbol{q}}^{*} \hat{b}_{\boldsymbol{q}}^{\dagger} \hat{c}_{\boldsymbol{q}+\boldsymbol{k} \uparrow}+u_{\boldsymbol{k}, \boldsymbol{q}}^{*} \hat{b}_{\boldsymbol{q}} \hat{c}_{\boldsymbol{q}-\boldsymbol{k} \downarrow}^{\dagger}\right) \\
& \quad+\frac{1}{N} \sum_{\boldsymbol{q}, \boldsymbol{q}^{\prime} \neq \mathbf{0}}\left(-v_{\boldsymbol{k}}^{*} \hat{c}_{\boldsymbol{k} \uparrow}+u_{\boldsymbol{k}, \boldsymbol{q}, \boldsymbol{q}^{\prime}}^{*} \hat{b}_{-\boldsymbol{q}}^{\dagger} \hat{b}_{-\boldsymbol{q}} \hat{\boldsymbol{q}}^{\prime} \hat{c}_{\boldsymbol{q}+\boldsymbol{q}^{\prime}+\boldsymbol{k} \uparrow}\right. \\
& \left.\quad+u_{\boldsymbol{k}, \boldsymbol{q}, \boldsymbol{q}^{\prime}}^{*} \hat{\boldsymbol{q}}_{\boldsymbol{q}} \hat{b}_{-\boldsymbol{q}^{\prime}}^{\dagger} c_{\boldsymbol{q}+\boldsymbol{q}^{\prime}-\boldsymbol{k} \downarrow}^{\dagger}\right)+\mathrm{O}\left(\hat{b}^{3}\right) .
\end{aligned}
$$

Scattering terms involving three and more pair operators are omitted because they are expected to be less probable. Equations (17), (18) must be supplemented by the corresponding ansatz for the boson operators $\hat{\tilde{b}}_{\boldsymbol{q}}^{(\dagger)}[31]$.

Let us remark that operators $\hat{b}_{\mathbf{0}}^{(\dagger)}$ referring to the $\mathrm{BE}$ condensed pairs simplify below $T_{\mathrm{c}}$ to complex numbers. Formally, we can thus interpret the coefficient $v_{\boldsymbol{k}}$ as resulting from the scattering on $\mathrm{BE}$ condensed bosons, i.e. $v_{\boldsymbol{k}, \mathbf{0}} \frac{\hat{b}_{0}}{\sqrt{N}}$. We use however $v_{\boldsymbol{k}}$ to have a clear correspondence of (17), (18) to the standard Bogoliubov transformation (11), (12).

To determine all the coefficients $u$ and $v$ we ought to fulfill the sum-rule (anticommutation relations between the new introduced operators). This will be done in the next subsection by means of the self-consistent flow equation procedure. Here, let us concentrate on the generic outcome of our proposal (17), (18). The single particle spectrum of fermions takes the following form:

$$
A(\boldsymbol{k}, \omega)=\left|u_{\boldsymbol{k}}\right|^{2} \delta\left(\omega-E_{\boldsymbol{k}}^{\mathrm{F}}\right)+\left|v_{\boldsymbol{k}}\right|^{2} \delta\left(\omega+E_{-\boldsymbol{k}}^{\mathrm{F}}\right)
$$

$$
\begin{aligned}
& +\frac{1}{N} \sum_{\boldsymbol{q} \neq \mathbf{0}}\left[\left|u_{\boldsymbol{k}, \boldsymbol{q}}\right|^{2} \alpha_{\boldsymbol{k}, \boldsymbol{q}}^{(1)} \delta\left(\omega-E_{\boldsymbol{q}+\boldsymbol{k}}^{\mathrm{F}}+E_{\boldsymbol{q}}^{\mathrm{B}}\right)\right. \\
& \left.+\left|v_{\boldsymbol{k}, \boldsymbol{q}}\right|^{2} \beta_{\boldsymbol{k}, \boldsymbol{q}}^{(1)} \delta\left(\omega+E_{\boldsymbol{q}-\boldsymbol{k}}^{\mathrm{F}}-E_{\boldsymbol{q}}^{\mathrm{B}}\right)\right] \\
& +N^{-2} \sum_{\boldsymbol{q}, \boldsymbol{q}^{\prime} \neq \mathbf{0}}\left[| u _ { \boldsymbol { k } , \boldsymbol { q } , \boldsymbol { q } ^ { \prime } } | ^ { 2 } \alpha _ { \boldsymbol { k } , \boldsymbol { q } , \boldsymbol { q } ^ { \prime } } ^ { ( 2 ) } \delta \left(\omega-E_{\boldsymbol{q}+\boldsymbol{q}^{\prime}+\boldsymbol{k}}^{\mathrm{F}}\right.\right. \\
& \left.+E_{\boldsymbol{q}}^{\mathrm{B}}+E_{\boldsymbol{q}}^{\mathrm{B}}\right)+\left|v_{\boldsymbol{k}, \boldsymbol{q}, \boldsymbol{q}^{\prime}}\right|^{2} \beta_{\boldsymbol{k}, \boldsymbol{q}, \boldsymbol{q}^{\prime}}^{(2)} \\
& \left.\delta\left(\omega+E_{\boldsymbol{q}+\boldsymbol{q}^{\prime}-\boldsymbol{k}}^{\mathrm{F}}-E_{\boldsymbol{q}}^{\mathrm{B}}-E_{\boldsymbol{q}^{\prime}}^{\mathrm{B}}\right)\right]+\mathrm{O}\left(\hat{b}^{3}\right) .
\end{aligned}
$$

Here $E_{\boldsymbol{k}}^{\mathrm{F}}$ and $E_{\boldsymbol{q}}^{\mathrm{B}}$ denote the effective excitation energies of fermions and bosons and coefficients $\alpha, \beta$ can be expressed by the occupancies of fermion and boson particles. Deep in a superconducting state fermions are characterized by the gaped Bogoliubov dispersion (while bosons develop the collective acoustic mode) therefore the spectral function contains some features due to scattering only outside the superconducting gap. Upon increasing temperature the number of thermally excited (finite momentum) bosons increases and this produces characteristic features in $A(\boldsymbol{k}, \omega)$. In particular, above $T_{\mathrm{c}}$ the term $\left|v_{\boldsymbol{k}}\right|^{2}$ completely vanishes but the low-lying bosons give the overdamped fermion branch located along the shadow part of quasiparticle branch $\omega \simeq-E_{\boldsymbol{k}}^{\mathrm{F}}$ through the contributions of $\left|v_{\boldsymbol{k}, \boldsymbol{q}}\right|^{2}$.

\subsection{The self-consistent procedure}

A fully self-consistent method for studying the model (13) can be constructed by the continuous canonical transformation procedure $\hat{H} \longrightarrow \mathrm{e}^{\hat{S}(l)} \hat{H} \mathrm{e}^{-\hat{S}(l)}$, where $l$ is some formal parameter [30]. The main idea is to eliminate the interaction part $g_{\boldsymbol{k}, \boldsymbol{q}}$ through a sequence of infinitesimal steps $l \rightarrow l+\delta l$. Proceeding along the lines of the renormalization group ( $\mathrm{RG}$ ) technique one starts from renormalizing the high energy sector and subsequently turns to the low energy sector, by latter we mean the fermion states close to $\mu$ and boson states near $2 \mu$.

Practically we start by setting $\hat{H}(l) \equiv \mathrm{e}^{\hat{S}(l)} \hat{H} \mathrm{e}^{-\hat{S}(l)}$, where $\hat{H}(0)$ corresponds to the initial Hamiltonian, and then construct the flow equation $\partial_{l} \hat{H}(l)=[\hat{\eta}(l), \hat{H}(l)]$ with the generating operator $\hat{\eta}(l) \equiv \partial_{l} \hat{S}(l)$. Following the original proposal of Wegner [30] we choose $\hat{\eta}(l)=$ $\left[\hat{H}_{0}(l), \hat{V}^{\mathrm{B}-\mathrm{F}}(l)\right]$, where $\hat{H}_{0}(l)$ denotes the total kinetic energy of fermions and bosons whereas $\hat{V}^{\mathrm{B}-\mathrm{F}}(l)$ stands for their interaction. From a straightforward algebra we obtain $\hat{\eta}(l)=-\frac{1}{\sqrt{N}} \sum_{\boldsymbol{k}, \boldsymbol{q}} \alpha_{\boldsymbol{k}, \boldsymbol{q}}(l)\left(b_{\boldsymbol{q}}^{\dagger} c_{\boldsymbol{q}-\boldsymbol{k} \downarrow} c_{\boldsymbol{k} \uparrow}-\right.$ H.c. $)$ with $\alpha_{\boldsymbol{k}, \boldsymbol{q}}(l)=\left(\varepsilon_{\boldsymbol{k}}(l)+\varepsilon_{\boldsymbol{q}-\boldsymbol{k}}(l)-E_{\boldsymbol{q}}(l)\right) g_{\boldsymbol{k}, \boldsymbol{q}}(l)$. We have previously shown [32] that such antihermitian operator $\hat{\eta}(l)$ guarantees an asymptotic disappearance of the boson-fermion coupling $\lim _{l \rightarrow \infty} g_{\boldsymbol{k}, \boldsymbol{q}}(l)=0$.

Applying this scheme to the BF Hamiltonian (13) we obtain the following set of coupled flow equations: $\partial_{l} g_{\boldsymbol{k}, \boldsymbol{q}}(l)=-\alpha_{\boldsymbol{k}, \boldsymbol{q}}^{2}(l) g_{\boldsymbol{k}, \boldsymbol{q}}(l)$, $\partial_{l} \varepsilon_{\boldsymbol{k}}(l)=\frac{2}{N} \sum_{\boldsymbol{q}} \alpha_{\boldsymbol{k}, \boldsymbol{q}}(l)\left|g_{\boldsymbol{k}, \boldsymbol{q}}(l)\right|^{2} n_{\boldsymbol{q}}^{\mathrm{B}}$ and $\partial_{l} E_{\boldsymbol{q}}(l)=$ $\frac{2}{N} \sum_{\boldsymbol{k}} \alpha_{\boldsymbol{k}-\boldsymbol{q}, \boldsymbol{k}}(l)\left|g_{\boldsymbol{k}-\boldsymbol{q}, \boldsymbol{k}}(l)\right|^{2}\left(-1+n_{\boldsymbol{k}-\boldsymbol{q} \downarrow}^{\mathrm{F}}+n_{\boldsymbol{k} \uparrow}^{\mathrm{F}}\right)[32]$. We have solved them numerically for the itinerant fermions and immobile bosons on the two-dimensional lattice. 
The fixed point values

$$
\lim _{l \rightarrow \infty} \varepsilon_{\boldsymbol{k}}(l) \equiv \tilde{\varepsilon}_{\boldsymbol{k}}, \quad \lim _{l \rightarrow \infty} E_{\boldsymbol{q}}(l) \equiv \tilde{E}_{\boldsymbol{q}}
$$

have shown the following properties: (a) below $T_{\mathrm{c}}$ the renormalized fermion dispersion $\tilde{\varepsilon}_{\boldsymbol{k}}$ develops a true gap at $\mu$ which evolves into a pseudogap for $T_{\mathrm{c}}<T$, (b) the effective boson dispersion $\tilde{E}_{\boldsymbol{q}}$ shows the long-wavelength Goldstone mode for $T<T_{\mathrm{c}}$ and its remnants are preserved even in the pseudogap state [17].

For a complete information about the fermion and boson spectra we must work out transformations for the individual operators $\hat{c}_{\boldsymbol{k} \sigma}^{(\dagger)}(l) \equiv \mathrm{e}^{\hat{S}(l)} \hat{c}_{\boldsymbol{k} \sigma}^{(\dagger)} \mathrm{e}^{-\hat{S}(l)}$ and $\hat{b}_{\boldsymbol{q}}^{(\dagger)}(l) \equiv \mathrm{e}^{\hat{S}(l)} \hat{b}_{\boldsymbol{q}}^{(\dagger)} \mathrm{e}^{-\hat{S}(l)}$ which is a rather difficult task because $\hat{S}(l)$ is not known explicitly. Since our primary interest is in the single particle fermion excitations, we focus on the flow equation $\partial_{l} \hat{c}_{\boldsymbol{k} \sigma}^{(\dagger)}(l)=\left[\hat{\eta}, \hat{c}_{\boldsymbol{k} \sigma}^{(\dagger)}(l)\right]$. The generating operator $\hat{\eta}(l)$ chosen according to Wegner's prescription [30] yields the following ansatz for fermion operators [17]:

$$
\begin{aligned}
& \hat{c}_{\boldsymbol{k} \uparrow}(l)=u_{\boldsymbol{k}}(l) \hat{c}_{\boldsymbol{k} \uparrow}+v_{\boldsymbol{k}}(l) \hat{c}_{-\boldsymbol{k} \downarrow}^{\dagger} \\
& \quad+\frac{1}{\sqrt{N}} \sum_{\boldsymbol{q} \neq \mathbf{0}}\left[u_{\boldsymbol{k}, \boldsymbol{q}}(l) \hat{b}_{\boldsymbol{q}}^{\dagger} \hat{c}_{\boldsymbol{q}+\boldsymbol{k} \uparrow}+v_{\boldsymbol{k}, \boldsymbol{q}}(l) \hat{b}_{\boldsymbol{q}} \hat{c}_{\boldsymbol{q}-\boldsymbol{k} \downarrow}^{\dagger}\right], \\
& \hat{c}_{-\boldsymbol{k} \downarrow}^{\dagger}(l)=-v_{\boldsymbol{k}}^{*}(l) \hat{c}_{\boldsymbol{k} \uparrow}+u_{\boldsymbol{k}}^{*}(l) \hat{c}_{-\boldsymbol{k} \downarrow}^{\dagger} \\
& +\frac{1}{\sqrt{N}} \sum_{\boldsymbol{q} \neq \mathbf{0}}\left[-v_{\boldsymbol{k}, \boldsymbol{q}}^{*}(l) \hat{b}_{\boldsymbol{q}}^{\dagger} \hat{c}_{\boldsymbol{q}+\boldsymbol{k} \uparrow}+u_{\boldsymbol{k}, \boldsymbol{q}}^{*}(l) \hat{b}_{\boldsymbol{q}} \hat{c}_{\boldsymbol{q}-\boldsymbol{k} \downarrow}^{\dagger}\right],
\end{aligned}
$$

where $u_{\boldsymbol{k}}(0)=1$ and all other coefficients are vanishing at $l=0$. The $l$-dependent coefficients must be determined from the following set of flow equations [17]:

$$
\begin{aligned}
& \partial_{l} u_{\boldsymbol{k}}(l)=\sqrt{n_{\boldsymbol{q}=\mathbf{0}}^{\mathrm{B}}} \alpha_{-\boldsymbol{k}, \mathbf{0}}(l) v_{\boldsymbol{k}}(l) \\
& +\frac{1}{N} \sum_{\boldsymbol{q} \neq \mathbf{0}} \alpha_{\boldsymbol{q}-\boldsymbol{k}, \boldsymbol{q}}(l)\left(n_{\boldsymbol{q}}^{\mathrm{B}}+n_{\boldsymbol{q}-\boldsymbol{k} \downarrow}^{\mathrm{F}}\right) v_{\boldsymbol{k}, \boldsymbol{q}}(l), \\
& \partial_{l} v_{\boldsymbol{k}}(l)=-\sqrt{n_{\boldsymbol{q}=\mathbf{0}}^{\mathrm{B}}} \alpha_{\boldsymbol{k}, \mathbf{0}}(l) u_{\boldsymbol{k}}(l) \\
& -\frac{1}{N} \sum_{\boldsymbol{q} \neq \mathbf{0}} \alpha_{\boldsymbol{k}, \boldsymbol{q}}(l)\left(n_{\boldsymbol{q}}^{\mathrm{B}}+n_{\boldsymbol{q}+\boldsymbol{k} \uparrow}^{\mathrm{F}}\right) u_{\boldsymbol{k}, \boldsymbol{q}}(l), \\
& \partial_{l} u_{\boldsymbol{k}, \boldsymbol{q}}=\alpha_{-\boldsymbol{k}, \boldsymbol{q}}(l) v_{\boldsymbol{k}}(l), \\
& \partial_{l} v_{\boldsymbol{k}, \boldsymbol{q}}=-\alpha_{\boldsymbol{k}, \boldsymbol{q}}(l) u_{\boldsymbol{k}}(l) .
\end{aligned}
$$

We have solved them numerically along with equations $\partial_{l} \varepsilon_{\boldsymbol{k}}(l), \partial_{l} E_{\boldsymbol{q}}(l), \partial_{l} g_{\boldsymbol{k}, \boldsymbol{q}}(l)$ for the 2-dimensional square lattice with the initial $(l=0)$ tight-binding dispersion

$$
\begin{gathered}
\varepsilon_{\boldsymbol{k}}=-2 t\left[\cos \left(k_{x} a\right)+\cos \left(k_{y} a\right)\right] \\
-4 t^{\prime} \cos \left(k_{x} a\right) \cos \left(k_{y} a\right)
\end{gathered}
$$

(we set $a \equiv 1$ ). We assumed the discrete boson energy $E_{\boldsymbol{q}}(0)=E_{0}$ and imposed $g_{\boldsymbol{k}, \boldsymbol{q}}(0)=g\left[\cos \left(k_{x} a\right)-\cos \left(k_{y} a\right)\right]$ in order to reproduce the $d$-wave symmetry of energy gap below $T_{\mathrm{c}}$. We have solved iteratively the coupled flow equations using the Runge-Kutta method for
$E_{0}(0)=0.2 t$ for the fixed total charge concentration $n_{\text {tot }}=2 \sum_{\boldsymbol{q}} n_{\boldsymbol{q}}^{\mathrm{B}}+\sum_{\boldsymbol{k}}\left(n_{\boldsymbol{k} \uparrow}^{\mathrm{F}}+n_{\boldsymbol{k} \downarrow}^{\mathrm{F}}\right)$. The number of fermions per lattice site $n^{\mathrm{F}}=1-p$ corresponds then roughly to $p \approx 0.1$ (see Fig. 1).

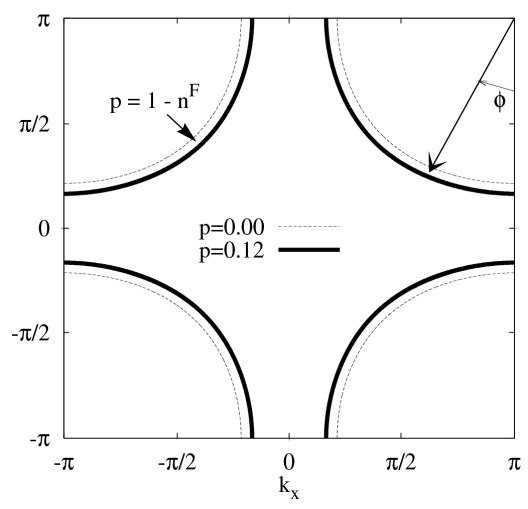

Fig. 1. Fermi surface of the noninteracting electrons for two-dimensional lattice described by (27) with the next-nearest neighbor hopping $t^{\prime} / t=-0.4$.

Our ansatz (21), (22) yields the effective single particle spectral function with the structure given in Eq. (19) neglecting terms of the order of $\mathrm{O}\left(\hat{b}^{2}\right)$ and the coefficients corresponding the fixed point values $l \rightarrow \infty$. Spectral function (19) indicates that besides the narrow peaks (long-lived states) there also forms a background of the damped (finite lifetime) states. If we neglect $u_{\boldsymbol{k}, \boldsymbol{q}}$ and $v_{\boldsymbol{k}, \boldsymbol{q}}$ then the flow equations (23), (24) simplify to $\partial_{l} u_{\boldsymbol{k}}(l)=\sqrt{n_{\boldsymbol{q}=\mathbf{0}}^{\mathrm{B}}} \alpha_{-\boldsymbol{k}, \mathbf{0}}(l) v_{\boldsymbol{k}}(l)$ and $\partial_{l} v_{\boldsymbol{k}}(l)=-\sqrt{n_{\boldsymbol{q}=\mathbf{0}}^{\mathrm{B}}} \alpha_{\boldsymbol{k}, \mathbf{0}}(l) u_{\boldsymbol{k}}(l)$ yielding the invariance $\left|v_{\boldsymbol{k}}(l)\right|^{2}+\left|v_{\boldsymbol{k}}(l)\right|^{2}=1$. By rewriting the first equation as $\int_{u_{\boldsymbol{k}}(0)=1}^{u_{\boldsymbol{k}}(\infty)=\tilde{u}_{\boldsymbol{k}}} \frac{d u_{\boldsymbol{k}}(l)}{\sqrt{1-\left|u_{\boldsymbol{k}}(l)\right|^{2}}}=\sqrt{n_{\boldsymbol{q}=\mathbf{0}}^{\mathrm{B}}} \int_{0}^{\infty} \alpha_{-\boldsymbol{k}, \mathbf{0}}(l) \mathrm{d} l$ we then right away reproduce the mean-field solution $\tilde{u}_{\boldsymbol{k}}, \tilde{v}_{\boldsymbol{k}}=\frac{1}{2}\left(1 \pm \frac{\varepsilon_{\boldsymbol{k}}-\mu}{\sqrt{\left(\varepsilon_{\boldsymbol{k}}-\mu\right)^{2}+n_{\mathbf{0}}^{\mathrm{B}}\left|g_{\boldsymbol{k}, \mathbf{o}}\right|^{2}}}\right)$. The effect of scattering on the finite momentum pairs affects (19) through the coefficients $\tilde{u}_{\boldsymbol{k}, \boldsymbol{q}}$ and $\tilde{v}_{\boldsymbol{k}, \boldsymbol{q}}$. We shall do it for $T>T_{\mathrm{c}}$.

\subsection{Bogoliubov quasiparticles above $T_{\mathrm{c}}$}

In the normal state the BE condensate does not exist $\left\langle\hat{b}_{\boldsymbol{q}=\mathbf{0}}\right\rangle=0$, therefore Eqs. (24), (25) analytically imply that $v_{\boldsymbol{k}}(l)=0=u_{\boldsymbol{k}, \boldsymbol{q}}(l)$. In consequence (21), (22) simplify to

$$
\begin{aligned}
& \hat{c}_{\boldsymbol{k} \uparrow}(l)=u_{\boldsymbol{k}}(l) \hat{c}_{\boldsymbol{k} \uparrow}+\frac{1}{\sqrt{N}} \sum_{\boldsymbol{q} \neq \mathbf{0}} v_{\boldsymbol{k}, \boldsymbol{q}}(l) \hat{b}_{\boldsymbol{q}} \hat{c}_{\boldsymbol{q}-\boldsymbol{k} \downarrow}^{\dagger}, \\
& \hat{c}_{-\boldsymbol{k} \downarrow}^{\dagger}(l)=-\frac{1}{\sqrt{N}} \sum_{\boldsymbol{q} \neq \mathbf{0}} v_{\boldsymbol{k}, \boldsymbol{q}}^{*}(l) \hat{b}_{\boldsymbol{q}}^{\dagger} \hat{c}_{\boldsymbol{q}+\boldsymbol{k} \uparrow}+u_{\boldsymbol{k}}^{*}(l) \hat{c}_{-\boldsymbol{k} \downarrow}^{\dagger},
\end{aligned}
$$

and under such conditions the lowest order estimation (but beyond the mean field level) of the spectral function (19) is given by 


$$
\begin{aligned}
& A(\boldsymbol{k}, \omega)=\left|\tilde{u}_{\boldsymbol{k}}\right|^{2} \delta\left(\omega+\mu-\tilde{\varepsilon}_{\boldsymbol{k}}\right) \\
& +\frac{1}{N} \sum_{\boldsymbol{q} \neq \mathbf{0}}\left(n_{\boldsymbol{q}}^{\mathrm{B}}+n_{\boldsymbol{q}-\boldsymbol{k} \downarrow}^{\mathrm{F}}\right)\left|\tilde{v}_{\boldsymbol{k}, \boldsymbol{q}}\right|^{2} \\
& \quad \times \delta\left(\omega-\mu+\tilde{\varepsilon}_{\boldsymbol{q}-\boldsymbol{k}}-\tilde{E}_{\boldsymbol{q}}\right)+\mathrm{O}\left(\hat{b}^{2}\right) .
\end{aligned}
$$

It consists of the delta peak corresponding to the long-lived quasiparticle state at $\tilde{\varepsilon}_{\boldsymbol{k}}-\mu$ whose spectral weight is $\left|\tilde{u}_{\boldsymbol{k}}^{2}\right|<1$ and the remaining weight shared between the damped fermion states.

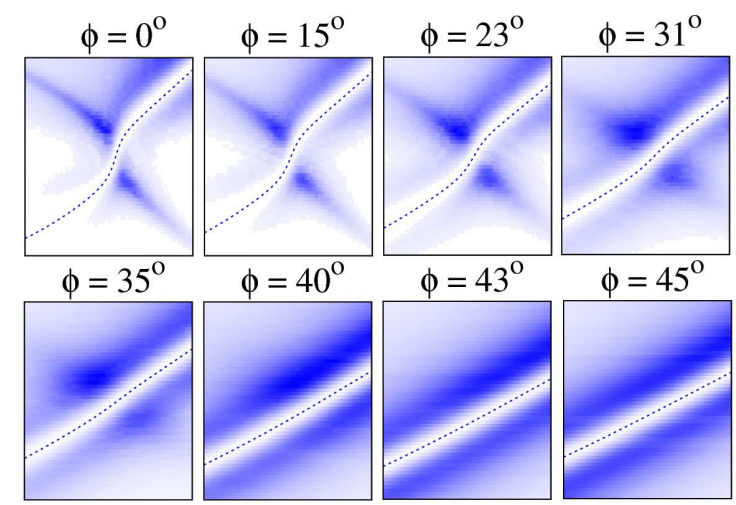

Fig. 2. The effective fermion spectrum in the pseudogap regime obtained for $T=0.007 D>T_{\mathrm{c}}$ with $D \equiv 8 t$. The thin dashed line shows the renormalized dispersion of the narrow quasiparticle at $\omega=\tilde{\varepsilon}_{\boldsymbol{k}}-\mu$ whereas the areas marked by colors illustrate the damped states. Notice that the Bogoliubov features are pronounced for the states aside the nodal direction.

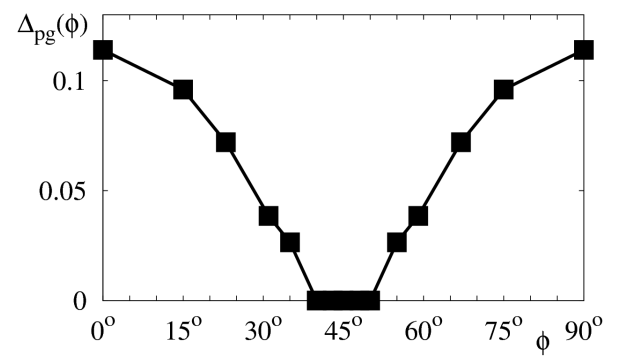

Fig. 3. Angular dependence of the pseudogap obtained above $T_{\mathrm{c}}$ for the same set of parameters as in Fig. 2.

Above $T_{\mathrm{c}}$ the quasiparticle peak continuously traverses the Fermi surface but approaching $T_{\mathrm{c}}$ its weight $\left|\tilde{u}_{\boldsymbol{k}}\right|^{2}$ is suppressed nearby $\boldsymbol{k}_{\mathrm{F}}$. We can notice in Fig. 2 that among the background states (which are almost insensitive to temperature) there is a certain fraction built around $\omega=-\left(\tilde{\varepsilon}_{\boldsymbol{k}}-\mu\right)$ for momenta located near the Fermi surface. Such branch of the excitations being a mirror reflection to the quasiparticle peak $\tilde{\varepsilon}_{\boldsymbol{k}}-\mu$ is the shadow branch of the Bogoliubov-type dispersion. Such quasiparticles, reminiscent of the superconducting state, have been indeed observed in the ARPES experiments of the Argonne [15] and the Swiss PSI [16] groups.
We have also evaluated the one-particle spectral function for momentum vectors $\boldsymbol{k}$ intersecting $\boldsymbol{k}_{\mathrm{F}}$ at various positions. The planar angle $\phi$ corresponds here to direction between the vector $\boldsymbol{k}-(\pi, \pi)$ and the line starting from $[\pi, \pi]$ to $[\pi, 0]$.

We have found that pseudogap disappears above $T_{\mathrm{c}}$ starting from the nodal points. As an illustration we show in Fig. 3 the angular variation of the pseudogap determined for $T=0.007 \mathrm{D}$. The partial closure of the $\phi$-dependent pseudogap leads to reconstruction of the Fermi surface pieces. We checked that length on these Fermi arcs is linearly increasing with respect to $T-T_{\mathrm{c}}$. A more detailed analysis of these properties will be discussed separately [33].

\section{Summary}

Besides the general remarks on the quantum superfluids for the boson and fermion systems we have analyzed in some detail the effect of superconducting fluctuations above the transition temperature assuming that the single fermions coexist and interact with the preformed pairs. Influence of fermion pairs on the single particle excitation spectrum was studied by means of the self-consistent RG-like method [30]. We have found that near $k_{\mathrm{F}}$ the renormalized dispersion $\tilde{\varepsilon}_{\boldsymbol{k}}$ leads to the pseudogap and additionally there appears a shadow part of the Bogoliubov-type dispersion near $\omega=-\left(\tilde{\varepsilon}_{\boldsymbol{k}}-\mu\right)$. This kind of features have been in fact seen in the recent ARPES measurements. It thus seems natural that absence of the large Fermi surface could be simply related to the pairing correlation, which the symmetry reasons are most effective in the antinodal areas.

\section{Acknowledgments}

This work is partly supported by the Ministry of Science and Education (Poland) under the grant NN202187833.

\section{References}

[1] W. Ketterle, M.W. Zwierlein, in: Proc. Int. School Phys. "Enrico Fermi", course CLXIV, Eds. M. Inguscio, W. Ketterle, C. Salomon, IOS Press, Amsterdam 2008, p. 95.

[2] S. Gorgini, L.P. Pitaevskii, S. Stringari, Rev. Mod. Phys. 80, 1215 (2008); I. Bloch, J. Dalibard, W. Zwerger, Rev. Mod. Phys. 80, 885 (2008).

[3] F. Dalfovo, S. Gorgini, L.P. Pitaevskii, S. Stringari, Rev. Mod. Phys. 71, 463 (1999).

[4] P. Pieri, G.C. Strinati, Phys. Rev. Lett. 91, 030401 (2003).

[5] Y.M. Bunkov, G.E. Volovik, JETP Lett. 89, 306 (2009); A.S. Borovik-Romanov, Yu.M. Bunkov, V.V. Dmitriev, Yu.M. Mukharskiy, D.A. Sergatskov, Phys. Rev. Lett. 62, 323 (1989).

[6] L.V. Butov, A.C. Gossard, D.S. Chemla, Nature 418, 751 (2002). 
[7] T. Fukuhara, S. Sugawa, Y. Takahashi, Phys. Rev. A 76, 051604 (2007); F. Dalfovo, L.P. Pitaevskii, S. Stringari, Rev. Mod. Phys. 71, 463 (1999).

[8] J.R. Abo-Shaer, C. Raman, J.M. Vogels, W. Ketterle, Science 292, 476 (2001).

[9] M. Albiez, R. Gati, J. Folling, S. Hunsmann, M. Cristiani, M.K. Oberhalter, Phys. Rev. Lett. 95, 010402 (2005); S. Levy, E. Lahoud, I. Shomroni, J. Steinhauer, Nature 449, 579 (2007).

[10] K.A. Chao, J. Spał, ek, A.M. Oleś, J. Phys. C 10 271L (1977); G. Baskaran, Z. Zou, P.W. Anderson, Solid State Commun. 63, 973 (1987).

[11] M.G. Alford, A. Schmitt, K. Rajagopal, T. Schofer, Rev. Mod. Phys. 80, 1455 (2008).

[12] V. Liu, F. Wilczek, Phys. Rev. Lett. 90, 047002 (2003).

[13] T. Domański, Phys. Rev. A 68, 013603 (2003).

[14] H. Matsui, T. Sato, T. Takahashi, S.-C. Wang, H.-B. Yang, H. Ding, T. Fujii, T. Watanabe, A. Matsuda, Phys. Rev. Lett. 90, 217002 (2003).

[15] A. Kanigel, U. Chatterjee, M. Randeria, M.R. Norman, G. Koren, K. Kadowaki, J. Campuzano, Phys. Rev. Lett. 1001, 137002 (2008).

[16] M. Shi, A. Bendounan, E. Razzoli, S. Rosenkranz, M.R. Norman, J.C. Campuzano, J. Chang, M. Mansson, Y. Sassa, T. Claesson, O. Tjernberg, L. Patthey, N. Momono, M. Oda, M. Ido, S. Guerrero, C. Mudry, J. Mesot, Eur. Phys. Lett. 88, 27008 (2009).

[17] T. Domański, J. Ranninger, Phys. Rev. Lett. 91 , 255301 (2003).

[18] P.A. Lee, N. Nagaosa, X.G. Wen, Rev. Mod. Phys. 78, 17 (2006); M. Eschrig, Adv. Phys. 55, 47 (2006); M. Norman, D. Pines, C. Kallin, Adv. Phys. 54, 715 (2005).

[19] O. Yuli, I. Asulin, Y. Kalchaim, G. Koren, O. Millo, Phys. Rev. Lett. 103, 197003 (2009).

[20] J. Orenstein, J. Corson, S. Oh, J.N. Eckstein, Ann. Phys. (Leipzig) 15, 596 (2006); Z.A. Xu, N.P. Ong, Y. Wang, T. Takeshita, S. Uchida, Nature 406, 486 (2000).

[21] V.J. Emery, S.A. Kivelson, Nature 374, 434 (1995).
[22] J. Ranninger, S. Robaszkiewicz, Physica B 135, 468 (1985); S. Robaszkiewicz, R. Micnas, J. Ranninger, Phys. Rev. B 36, 180 (1987).

[23] K. McElroy, Nature Phys. 2, 441 (2006).

[24] R. Micnas, J. Ranninger, S. Robaszkiewicz, Rev. Mod. Phys. 62, 113 (1990); R. Friedberg, T.D. Lee, Phys. Rev. B 40, 423 (1989); J. Ranninger, J.M. Robin, Physica C 253, 279 (1995).

[25] Z. Tesanović, Nature Phys. 4, 408 (2008); J.A. Wilson, Philos. Mag. 84, 2183 (2004); E. Altman, A. Auerbach, Phys. Rev. B 65, 104508 (2002); M. Franz, Z. Tesanovic, Phys. Rev. Lett. 87, 257003 (2001); C. Lannert, M.P.A. Fisher, T. Senthil, Phys. Rev. B 64, 014518 (2001); V.B. Geshkenbein, L.B. Ioffe, A.I. Larkin, Phys. Rev. B 55, 3173 (1997).

[26] P.W. Anderson, P.A. Lee, M. Randeria, T.M. Rice, F.C. Zhang, J. Phys., Condens. Matter 16, R755 (2005).

[27] Q. Chin, J. Stajic, S. Tan, K. Levin, Phys. Rep. 412, 1 (2005).

[28] T. Kostyrko, J. Ranninger, Phys. Rev. B 54, 13105 (1996); Y. Ohashi, A. Griffin, Phys. Rev. Lett. 89 , 130402 (2002).

[29] C.A.R. Sa, de Melo, M. Randeria, J. Engelbrecht, Phys. Rev. Lett. 71, 3202 (1993); H.-J. Kwon, A.T. Dorsey, Phys. Rev. B 59, 6438 (1999); M.Y. Veillette, D. Sheehy, L. Radzihovsky, Phys. Rev. A 75, 043614 (2007).

[30] F. Wegner, Ann. Phys. 3, 77 (1994); S. Kehrein, Flow Equation Approach to Many Particle Systems, Springer Tracts in Modern Physics, Vol. 217, Berlin 2006.

[31] T. Domański, J. Ranninger, Phys. Rev. B 70, 184503 (2004).

[32] T. Domański, J. Ranninger, Phys. Rev. B 63, 134505 (2001).

[33] J. Ranninger, T. Domański, Phys. Rev. B 81, 014514 (2010) 